\title{
Management and surgical treatment of parathyroid crisis secondary to parathyroid tumors: report of four cases
}

This article was published in the following Dove Press journal:

International Medical Case Reports Journal

I2 August 201 I

Number of times this article has been viewed

\author{
Shakil Ameerudden \\ Xianghui He \\ Department of General Surgery, \\ Tianjin Medical University General \\ Hospital, Tianjin, China
}

\begin{abstract}
Parathyroid crisis, also known as a parathyroid storm, is a rare and serious complication of primary hyperparathyroidism. Four cases are reported here in which patients presented to hospital with general complaints due to hypercalcemia secondary to hyperparathyroidism. Blood test results upon admission showed high levels of serum calcium and parathyroid hormone, and medical treatment initiated to lower the calcium level was ineffective. After relevant investigations, each patient underwent surgical exploration of the parathyroid glands, followed by excision of a pathological parathyroid tumor. There was a prompt decrease in parathyroid hormone level immediately after surgery. Histology reports revealed that patients had parathyroid adenoma. All patients recovered after surgery, with serum calcium levels restored back to normal and with resolution of all symptoms of hypercalcemia. This report illustrates how often this disease is initially misdiagnosed, and how prompt appropriate surgical treatment provides the best outcome for the patient.
\end{abstract}

Keywords: parathyroid crisis, hypercalcemia, hyperparathyroidism, hypercalcemic crisis, parathyroid adenoma

\section{Introduction}

Primary hyperparathyroidism (PHPT) is a disease characterized by hypercalcemia attributable to autonomous overproduction of parathyroid hormone. Parathyroid crisis is a rare and potentially fatal complication of PHPT in which patients develop severe hypercalcemia with signs and symptoms of multiple organ dysfunction. PHPT is a relatively common disorder affecting 1 in 500 women and 1 in 2000 men over 40 years of age. The best estimate of incidence is 22 per 100,000 per year, and prevalence is approximately one per $1000 .^{1}$ PHPT is caused by a single parathyroid adenoma in about $80 \%-85 \%$ of cases. The rest of the cases of PHPT are due to multiple gland hyperplasia affecting all parathyroid glands in about $10 \%$, double adenomas in $4 \%$, and parathyroid carcinoma in $1 \%$.

Serum levels of calcium are closely regulated, with a normal total calcium of $2.2-2.6 \mathrm{mmol} / \mathrm{L}(9-10.5 \mathrm{mg} / \mathrm{dL})$ and a normal ionized calcium of $1.1-1.4 \mathrm{mmol} / \mathrm{L}$ $(4.5-5.6 \mathrm{mg} / \mathrm{dL})$. Patients with calcium levels of $2.6-3.0 \mathrm{mmol} / \mathrm{L}$ may be asymptomatic. When the serum calcium level rises above this stage, multisystem clinical manifestations become apparent. However, in $80 \%$ of patients with hyperparathyroidism, the symptoms of hypercalcemia are mild or are not notable at the time of discovery. As a result, this disease is often misdiagnosed or overlooked completely, yet it can be effectively treated if recognized and treated in time. Therefore, it is very important that initial evaluation of a patient with general symptoms consistent with hypercalcemia should include a careful 
history and physical examination, focusing on the clinical manifestations of hypercalcemia, risk factors for malignancy, and causative medications. Family history of hypercalcemiaassociated conditions should also be taken in consideration. These patients must be subsequently tested for elevated serum calcium and parathyroid hormone levels. The diagnosis of hyperparathyroidism is usually first suspected because of the finding of an elevated serum calcium concentration. If hypercalcemia is confirmed on a repeat sample, hyperparathyroidism should be considered. The diagnosis of primary hyperparathyroidism is then usually made by finding a frankly elevated parathyroid hormone concentration or which is inappropriately elevated given the level of hypercalcemia. Normal values of parathyroid hormone are $10-55 \mathrm{ng} / \mathrm{L}$.

Surgical removal of a solitary parathyroid tumor or subtotal resection of all pathologic parathyroid tissue in patients with hyperparathyroidism results in normalization of parathyroid hormone secretion, normalization of serum calcium levels, and a durable cure. This report also outlines the difficulty of an initial diagnosis due to atypical presentations, the relevance of medical stabilization through fluid therapy and specific medications, and the importance of imaging prior to surgery. Furthermore, it shows clear evidence that parathyroidectomy is the most efficient curative therapy for PHPT. It is both safe and cost-effective, and in optimum conditions the cure rate is reported as being as high as $98 \%{ }^{2}$ We report four cases where patients presented with hypercalcemic symptoms secondary to hyperparathyroidism due to parathyroid adenomas.

\section{Case report I}

A 53-year-old male patient presented to the Department of Endocrinology at our hospital complaining of fatigue, generalized weakness, body discomfort, lack of appetite, and nausea for one week. For the few days before admission, the patient was drowsy and confused. Blood test reports showed a serum calcium level of $4.12 \mathrm{mmol} / \mathrm{L}$ and parathyroid hormone level of $491 \mathrm{ng} / \mathrm{L}$. The thyroid ultrasound report showed no abnormality of the bilateral parathyroid area. Technetium-99m ( $\left.{ }^{99 \mathrm{~m}} \mathrm{Tc}\right)$ sestamibi imaging revealed abnormal uptake of tracer in the lower aspect of the right lobe of the thyroid gland, suggestive of a hyperfunctioning parathyroid gland. The initial management plan on admission included aggressive fluid resuscitation. Calcitonin and alendronate sodium were also given to reduce the serum calcium. On day 2 , the patient was still drowsy and confused, and his general condition showed no improvement. The serum calcium level was only slightly reduced to $3.52 \mathrm{mmol} / \mathrm{L}$. The patient was then referred to the Department of General Surgery for emergency surgical treatment. A friable reddish-brown mass of about $3 \mathrm{~cm} \times 4 \mathrm{~cm}$ in size was excised below the right thyroid gland. The pathology report confirmed this resected right inferior mass to be hypercellular parathyroid tissue consistent with parathyroid adenoma. After surgery, the patient's condition improved gradually over the next 28 days, resulting in a serum calcium level of $2.15 \mathrm{mmol} / \mathrm{L}$ and an ionized serum calcium level of $1.1 \mathrm{mmol} / \mathrm{L}$. The patient's condition became satisfactory and he was discharged, with close follow-up over the following six months. At the oneweek follow-up appointment, the patient had no complaints and showed no symptoms associated with hypercalcemia. The serum calcium level at this time was $2.32 \mathrm{mmol} / \mathrm{L}$ and the parathyroid hormone level was $12.4 \mathrm{ng} / \mathrm{L}$. After two months, the serum calcium level was $2.25 \mathrm{mmol} / \mathrm{L}$. Upon review four months later, the patient was still normocalcemic with a total serum calcium level $2.32 \mathrm{mmol} / \mathrm{L}$ and a normal parathyroid hormone level of $21 \mathrm{ng} / \mathrm{L}$.

\section{Case report 2}

A 56-year-old female patient attended the Department of Orthopedics at our hospital with a history of knee and hip pain for more than five years, and of increasing intensity during the previous six months. The patient also complained of nausea, fatigue, and several episodes of vomiting during the previous week. She was admitted with a diagnosis of osteoporosis. Blood tests revealed a serum calcium level of $3.89 \mathrm{mmol} / \mathrm{L}$ and a parathyroid hormone level of $274 \mathrm{ng} / \mathrm{L}$. The initial management plan included intensive fluid therapy with normal saline. Salmon calcitonin together with pamidronate disodium was also given to decrease serum calcium. After five days, the serum calcium was still at $2.88 \mathrm{mmol} / \mathrm{L}$. Ultrasound and computed tomography (CT) scan of the thyroid glands both indicated a posterior right lobe cystic mass, and the ${ }^{99 \mathrm{~m}} \mathrm{Tc}$ sestamibi imaging indicated a hyperfunctioning right lower parathyroid lesion. Seven days after admission and medical treatment, the serum calcium level was still raised at $3.6 \mathrm{mmol} / \mathrm{L}$. The patient was then referred to the Department of General Surgery for emergency surgical treatment. A $1 \mathrm{~cm} \times 1 \mathrm{~cm} \times 1 \mathrm{~cm}$ friable mass on the posterior inferior aspect of the right thyroid lobe was excised during surgery. The patient also underwent right hemithyroidectomy after goitrous nodular changes were observed in the right thyroid gland. The frozen section pathology report of the resected right thyroid gland was suggestive of nodular goiter, and the excised mass indicated parathyroid adenoma with cystic changes. On the first postoperative day, the total 
serum calcium was $2.14 \mathrm{mmol} / \mathrm{L}$ and parathyroid hormone was $0.81 \mathrm{ng} / \mathrm{L}$. Three days later, the patient complained of body numbness and mouth twitching due to postoperative reactive hypocalcemia. Consequently, calcium gluconate $10 \%$ and other calcium supplements were given to the patient, after which the serum calcium level returned back to normal. The patient's condition eventually improved and she was discharged with regular follow-up for six months.

\section{Case report 3}

A 67-year-old female patient attended the Department of Gastroenterology at our hospital complaining of abdominal pain, intermittent nausea, and vomiting for one month. The patient had a past medical history of coronary heart disease and hypokalemia. Upon admission, medical treatment provided no relief to the patient and there were no signs of improvement of the patient's condition over the following two days. Blood test reports showed a serum potassium level of $2.7 \mathrm{mmol} / \mathrm{L}$, a serum calcium level of $4.16 \mathrm{mmol} / \mathrm{L}$, and a parathyroid hormone level of $164 \mathrm{ng} / \mathrm{L}$. Thyroid CT scan reported a soft tissue high density mass posterior to the right thyroid lobe. The ${ }^{99 \mathrm{~m}} \mathrm{Tc}$ sestamibi report indicated that the lower pole of the right thyroid lobe showed signs of abnormality. The report was also suggestive of general hyperactive bone metabolism, consistent with metabolic bone disease. The patient was then admitted to the Department of General Surgery for surgical treatment and fluid therapy was immediately started. The total serum calcium level prior to surgery was $3.91 \mathrm{mmol} / \mathrm{L}$. At the lower pole of the right thyroid, a tumor $2 \mathrm{~cm}$ in diameter was observed. Prior to tumor resection, the parathyroid hormone level was $141 \mathrm{ng} / \mathrm{L}$, and 10 minutes after tumor resection, the parathyroid hormone level dropped to $32.1 \mathrm{ng} / \mathrm{L}$. The frozen section pathology report was suggestive of parathyroid adenoma. Five days after surgery, the calcium level was $1.98 \mathrm{mmol} / \mathrm{L}$, and the patient complained of numbness and mouth twitching. Calcium supplements were therefore prescribed to increase the serum calcium. The patient gradually recovered, with laboratory results showing a normal serum calcium level. She was discharged 10 days later, with regular follow-up over six months to recheck the calcium level.

\section{Case report 4}

A 52-year-old male patient attended hospital complaining of poor appetite, nausea, weakness, and fatigue for the previous 20 days. The patient had no relevant past medical or surgical history. He was admitted to the Department of Medicine at our hospital, and supportive medical treatment was started immediately with fluid therapy. The patient's general condition showed no improvement over the next two days. Blood results showed a serum calcium of $4.52 \mathrm{mmol} / \mathrm{L}$ and a parathyroid hormone level of $254 \mathrm{ng} / \mathrm{L}$. The CT scan report of the thyroid indicated irregular low-density soft tissue nodules posterior to the upper right thyroid lobe. ${ }^{99 \mathrm{~m}} \mathrm{Tc}$ sestamibi imaging showed abnormal tracer concentrations at the upper pole of the right thyroid lobe, suggestive of a hyperfunctioning right upper parathyroid gland. Calcitonin was given, but there was still no significant improvement in the patient's condition, and a serum calcium recheck was $4.32 \mathrm{mmol} / \mathrm{L}$. The patient was admitted to the Department of Surgery, and bilateral parathyroid surgical exploration showed a reddish-brown $2.5 \mathrm{~cm} \times 2.5 \mathrm{~cm} \times 1.5 \mathrm{~cm}$ encapsulated tumor at the right upper thyroid lobe. The tumor was excised and the frozen section sent for analysis. The pathology report indicated parathyroid adenoma. On the first postoperative day, serum calcium was $2.32 \mathrm{mmol} / \mathrm{L}$, was $2.15 \mathrm{mmol} / \mathrm{L}$ three days later, and was $2.14 \mathrm{mmol} / \mathrm{L}$ one week later. Parathyroid hormone was $12.3 \mathrm{ng} / \mathrm{L}$. The patient's condition improved gradually over the next two weeks and he was eventually discharged, with regular follow-up over the next six months.

\section{Discussion Clinical features}

The mnemonic "stones, bones, abdominal moans, and psychic groans" is commonly used to recall the signs and symptoms of hypercalcemia. Neuromuscular effects include fatigue, lethargy, muscle weakness, impaired concentration, confusion, and stupor. Severe elevations in calcium levels may even cause coma. Nausea, vomiting, abdominal pain, anorexia, and constipation are among the common gastrointestinal manifestations. Pancreatitis and peptic ulcer disease secondary to hypercalcemia may sometimes complicate the picture further. Renal involvement is a frequent feature in severe cases of hypercalcemia, and renal colic as a consequence of nephrolithiasis is also an important presenting complaint. Other renal effects are polydipsia and polyuria resulting from nephrogenic diabetes insipidus. Cardiovascular effects include hypertension, vascular calcification, and a shortened QT interval on the electrocardiogram. Skeletal involvement is common, with features of hyperparathyroid bone disease resulting in bone pain, osteoporosis, and pathological fractures. Radiological hallmarks include osteitis fibrosa cystica and subperiosteal bone resorption. Symptoms of hypercalcemia are usually nonspecific, and are related to the severity and rate of change of the serum 
calcium level. Symptoms are more severe with acute changes than with chronic calcium level elevation.

\section{Diagnosis}

Hypercalcemic crisis is characterized by severe hypercalcemia, usually with a calcium level greater than $3.5 \mathrm{mmol} / \mathrm{L}$, with associated signs and symptoms involving multiple organ systems. Patients may initially show mild symptoms, but if the disease is still untreated, they may eventually develop metabolic encephalopathy, renal insufficiency, gastrointestinal symptoms, and cardiac dysrhythmia. ${ }^{3}$ Primary hyperparathyroidism is one of the most common causes of hypercalcemia and should be considered in any individual with an elevated calcium level. ${ }^{4}$ An interesting fact is that all the four cases reported here presented to hospital with mild and general complaints but with abnormally high serum calcium levels. The incidence and prevalence of PHPT globally are similar to that of the US and European countries, but the presentation varies greatly. In the US and Europe, most patients $(80 \%)$ present with asymptomatic disease. However, it is reported that in resource-poor nations most $(>80 \%)$ patients present with symptoms ${ }^{5}$ and often with relatively high serum calcium levels. This interesting fact can be observed in our case reports, and may be explained by frequent misdiagnosis or late referral to hospital for treatment. All four patients were admitted to different hospital departments, thus underscoring the difficulty in the initial diagnosis prior to laboratory results and imaging. However, early and timely diagnosis followed by prompt surgical treatment would be the key to effective treatment and definitely safeguard the patient against more severe complications of hypercalcemia.

It is also interesting to note that all of the patients reported here showed some clinical features and relatively high serum calcium levels that are generally associated with malignancy. Parathyroid carcinoma is a rare disease that accounts for only $1 \%-3 \%$ of cases of primary hyperparathyroidism, with the majority of cases caused by parathyroid adenomas. ${ }^{6}$ A parathyroid carcinoma is usually difficult to diagnose preoperatively and is not conclusively identified intraoperatively. Preoperative suspicion may be raised if the patient is young with a palpable neck mass and showing signs of recurrent laryngeal nerve palsy, or hyperparathyroid, renal, or skeletal disease, and has extremely high calcium or parathyroid hormone levels. ${ }^{7}$ Intraoperatively, the surgeon may suspect carcinoma if the gland is large, pale, and abuts the thyroid lobe, with the presence of dense adhesions. However, the surgical findings may also be the same for parathyroid adenoma.
In practice, all the features mentioned do not always appear and sometimes they are also consistent with benign parathyroid disease, as in our four cases. However, recognition is vital because a more aggressive surgical approach is essential for the treatment of patients with parathyroid carcinomas. The most effective treatment for parathyroid carcinoma is surgical, and consists of en bloc resection of the tumor, including removal of any enlarged or abnormal lymph nodes and any adherent tissue. ${ }^{8}$ Rupture of the capsule should be carefully avoided, because this may cause local seeding. Ipsilateral thyroid lobectomy is usually mandatory in such cases of malignancy.

Histological diagnosis to distinguish parathyroid carcinoma from the more frequently seen adenoma and hyperplasia can be very difficult. The Schantz and Castleman histological criteria ${ }^{9}$ can be helpful for differentiation of parathyroid carcinoma from adenoma. Cytologic features of carcinoma include atypical mitotic figures, nuclear pleomorphism, and nuclear enlargement. Typical features of parathyroid carcinoma also include the presence of a thick fibrous capsule with dense fibrous septa extending and dividing the gland and a trabecular or rosette-like tumor cell arrangement. The criteria upon which a definitive diagnosis of parathyroid carcinoma can be made include capsular invasion, vascular invasion, or local invasion into adjacent soft tissues, and lymph node or distant metastases. However, differentiation between carcinoma and degenerative changes in a hyperplastic or adenomatous parathyroid gland can prove to be even more difficult due to subsequent fibrosis and invasion of the capsule. ${ }^{10}$ An atypical adenoma may also show some histological features associated with parathyroid carcinoma but not sufficient for definite diagnosis. It is to be highlighted that, although all of our patients presented with abnormally high serum calcium levels and some clinical signs of malignancy, the histopathology reports after surgery did not mention any features of parathyroid carcinoma. A practical approach for making a histologic distinction between parathyroid carcinoma and adenoma is outlined in Table 2. ${ }^{11}$

The total serum calcium blood results are often inaccurate, and at least two positive results are required. Ionized serum calcium level is a more accurate diagnostic result. The parathyroid hormone level is also a very reliable laboratory diagnostic criterion, and combined with the serum calcium level, shows direct evidence of hyperparathyroidism. Rapid comparison of intraoperative parathyroid hormone levels with preoperative levels is very useful to confirm that there is no residual presence of multiple adenomas, and thus avoiding the omission of multiple lesions. It is particularly useful in 
Table I Effect of medical treatment on serum calcium levels

\begin{tabular}{lllll}
\hline Case & $\begin{array}{l}\text { Total serum calcium } \\
\text { on admission }(\mathbf{m m o l} / \mathbf{L})\end{array}$ & $\begin{array}{l}\text { Medical } \\
\text { treatment }\end{array}$ & $\begin{array}{l}\text { Preoperative total } \\
\text { serum calcium (mmol/L) }\end{array}$ & $\begin{array}{l}\text { Postoperative total } \\
\text { serum calcium (mmol/L) }\end{array}$ \\
\hline I & 4.12 & $\begin{array}{l}\text { Normal saline infusion, calcitonin, } \\
\text { alendronate }\end{array}$ & 3.52 & 2.15 \\
2 & 3.89 & $\begin{array}{l}\text { Normal saline infusion, calcitonin, } \\
\text { pamidronate }\end{array}$ & 3.60 & 2.14 \\
3 & 4.16 & Normal saline infusion & 3.91 & 1.98 \\
4 & 4.52 & Normal saline infusion, calcitonin & 4.32 & 2.32 \\
\hline
\end{tabular}

the setting of negative sestamibi scanning and in complex multigland disease cases. ${ }^{12}$ Parathyroid hormone, which is produced by the parathyroid glands, is cleared from the blood stream very quickly. Within 10 minutes of removing all hyperactive parathyroid tissue, parathyroid hormone levels should fall by more than half. Therefore, blood is drawn during surgery for parathyroid hormone assays before and after excision of a hyperfunctioning gland. A decrease of more than $50 \%$ from the baseline parathyroid hormone value at 10 minutes after resection is suggestive of a single site of primary hyperparathyroidism, generally a single parathyroid adenoma. If the parathyroid hormone levels do not drop by $50 \%$ then there is likely additional abnormal tissue, and the surgeon will proceed to identify and evaluate each of the remaining glands. In those cases, double parathyroid gland adenomas or four-gland hyperplasia is likely, and the patient usually must undergo bilateral neck exploration. ${ }^{13}$ New surgical approach combining preoperative localization studies and intraoperative parathyroid hormone monitoring have eliminated the most common causes of parathyroidectomy failure and has significantly decreased the operative failure rate. ${ }^{14}$
The noninvasive imaging modalities commonly used in patients with primary hyperparathyroidism include ${ }^{99 \mathrm{~m}}$ Tc sestamibi imaging, ultrasonography, CT scanning, and magnetic resonance imaging. The accuracy of those radiologic modalities is variable. They are not required for the diagnosis of hyperparathyroidism, but serve mainly as guides for surgical strategy. The results of multiple comparative studies have shown the superiority of scintigraphy for preoperative localization. The diagnostic utility of ${ }^{99 \mathrm{~m}} \mathrm{Tc}$ sestamibi imaging equals or exceeds other noninvasive, nonscintigraphic imaging strategies, including high-resolution ultrasound, CT, and magnetic resonance imaging. ${ }^{15}$ One meta-analysis of the medical literature reported that the overall sensitivity of dual-phase ${ }^{99 \mathrm{~m}} \mathrm{Tc}$ sestamibi in comparison with high-resolution ultrasonography was $88 \%$ versus $78 \%$ for single adenomas and $30 \%$ versus $16 \%$ for double adenomas. ${ }^{16}$ Combined ultrasound and ${ }^{99 \mathrm{~m}} \mathrm{Tc}$ sestamibi are reported to have increased sensitivity for the preoperative localization of parathyroid adenomas. ${ }^{17,18}$ Contrast materialenhanced $\mathrm{CT}$ and magnetic resonance imaging may also be effective for localizing parathyroid adenomas. Thin-section contrast-enhanced CT is reported to have a sensitivity ranging

Table 2 Histologic criteria for diagnosis of malignancy in parathyroid neoplasm

\begin{tabular}{ll}
\hline Absolute criteria of malignancy & Features associated with malignancy \\
\hline $\begin{array}{l}\text { Presence of any one of the following criteria is sufficient } \\
\text { for a diagnosis of malignancy }\end{array}$ & $\begin{array}{l}\text { In the absence of the absolute criteria, at least 2, preferably } 3 \text { or more, } \\
\text { of the following features have to be present in order to establish a } \\
\text { Invasion into surrounding tissues: }\end{array}$ \\
$\begin{array}{l}\text { diagnosis of malignancy } \\
\text { Capsular invasion }\end{array}$ \\
- esophagus & Vascular invasion \\
- nerves or & Readily identifiable mitotic figures $(>5 / I 0$ high-powered field) \\
- soft tissues & Broad intratumoral fibrous bands splitting the parenchyma \\
Histologically documented regional or distant metastasis & and separating expansible nodules \\
& Coagulative tumor necrosis (has to be distinguished from \\
& infarction, which can occur in parathyroid adenoma) \\
& Diffuse sheet-like monotonous small cells with high \\
nuclear/cytoplasmic ratio
\end{tabular}

Note: This article was published in Seminars in Diagnostic Pathology. Chan JK, Tsang WY. Endocrine malignancies that may mimic benign lesions. Semin Diagn Pathol. 1995;12:45-63. Copyright@ Elsevier 1995." 
from $46 \%$ to $87 \%$ for the detection of parathyroid adenomas, whereas the reported sensitivity of magnetic resonance imaging ranges from $65 \%$ to $80 \% .{ }^{19}$ However, in practice, they are used less commonly for preoperative localization than for the detection of a suspected ectopic mediastinal parathyroid adenoma in case of failed parathyroidectomy. ${ }^{20}$ ${ }^{99 \mathrm{~m}} \mathrm{Tc}$ sestamibi single photon emission CT can also nowadays be used in combination with x-ray-based CT for the localization of parathyroid adenomas in three dimensions, thus providing even more accurate anatomic information to the surgeon.

\section{Management}

The need for treatment of hypercalcemia depends on the degree of hypercalcemia and the presence or absence of clinical symptoms. Asymptomatic patients with a total serum calcium level less than $3.0 \mathrm{mmol} / \mathrm{L}$ generally do not benefit from normalization of their serum calcium levels. Asymptomatic patients with moderate calcium elevations of $3.0-3.5 \mathrm{mmol} / \mathrm{L}$ require only adequate hydration, whereas in patients showing symptoms consistent with hypercalcemia, aggressive treatment is necessary for correction of hypercalcemia. Patients with calcium levels higher than $3.5 \mathrm{mmol} / \mathrm{L}$ should be treated urgently and aggressively, regardless of symptoms. ${ }^{21}$

The initial therapy of any patients with moderate to severe hypercalcemia should be intravenous hydration with isotonic saline. Intravenous administration of isotonic saline is the first and most vital step in the management of hypercalcemia. Hydration helps decrease the calcium level through dilution. The expansion of extracellular volume also increases the renal calcium clearance. The rate of fluid therapy is based upon the degree of hypercalcemia, the severity of dehydration, and the ability of the patient to tolerate rehydration. Intravenous rehydration is normally best achieved using normal saline 2-4 L daily for 1-3 days, but it is to be noted that hydration is ineffective in patients with kidney failure because diuresis is impossible. Patients with renal failure or congestive heart disease who are at risk of fluid overload would require dialysis to correct hypercalcemia. ${ }^{22}$ A loop diuretic can be used in low dosages to lower the serum calcium level further if necessary. However, diuretics should be started only when the intravascular volume has been restored, with a urinary flow of $200-250 \mathrm{~mL} /$ hour. Intravenous furosemide $10-20 \mathrm{mg}$ is a commonly used diuretic for this purpose. Diuretics may also prevent volume overload during therapy.

Inhibition of the bone resorption process results in lowering of the serum calcium level. Drugs used for this purpose include calcitonin and bisphosphonates. They may be used in critical patients with moderate to severe hypercalcemia, where hydration therapy is not being effective or contraindicated, to alleviate the clinical manifestations of hypercalcemic disorders. Calcitonin can be given subcutaneously or intramuscularly every 12 hours at a dosage of $4 \mathrm{IU} / \mathrm{kg}$. Its action is rapid over 4-6 hours but it has a short duration of effect. Calcitonin can be used in patients with moderate to severe hypercalcemia. However, because of the short duration of action, other more potent but slower-acting agents are usually started in patients with severe hypercalcemia. Another drawback of calcitonin is that it is effective in only $60 \%-70 \%$ of patients and, furthermore, patients tend to develop tolerance to the calcium-lowering effect. As observed in Table 1, the first and second case reports described here were initially both treated with calcitonin. However, the outcome was only limited reduction in the total serum calcium level, with no improvement in general condition.

Bisphosphonates are a group of medications that accumulate in bone and powerfully inhibit osteoclast-mediated bone resorption. Biphosphonates are used effectively in cases of severe hypercalcemia mainly resulting from parathyroid malignancy. ${ }^{23}$ However, peak effects do not occur until 48-72 hours after infusion, and the duration of effect is usually several weeks, therefore considerably increasing the risk of hypocalcemia. An interesting observation is that two of our four patients were treated with biphosphonates prior to surgery, but all four patients had mild postoperative hypocalcemia, as outlined in Table 1. It is unclear whether the cause of hypocalcemia was due to the effects of biphosphonates or to the "hungry bone syndrome", ${ }^{24}$ where the sudden postoperative withdrawal of parathyroid hormone induces abrupt cessation of osteoclastic bone resorption without affecting osteoblastic activity. Typically, patients having extreme elevations of serum calcium preoperatively are more at risk of developing symptomatic postoperative hypocalcemia, as observed in our case reports. Pamidronate is used most commonly. It is given by intravenous infusion over 4-24 hours at a dosage of 30-90 mg. ${ }^{25}$ Because of the lag in onset of effect, bisphosphonates are often combined with faster-acting therapeutic modalities, such as intravenous saline infusion and calcitonin injections. However, caution must be exercised when using bisphosphonates ${ }^{26}$ in patients with renal impairment. Other antiresorptive agents that are used occasionally include plicamycin and gallium nitrate. Initial medical stabilization and optimization is an important step prior to surgery. Rehydration, calciuresis, and bisphosphonate therapy are effective for correcting life-threatening 
manifestations of hyperparathyroid crisis, and preparing the patient for forthcoming surgery. ${ }^{27}$ However, it is also to be noted that while preoperative medical optimization can be successful, there are reports where medical management has failed, and the disease has only responded to definitive surgical management. ${ }^{28}$

Parathyroid surgery remains the single most effective treatment option in hyperparathyroidism and requires removal of all abnormal parathyroid tissue. Traditionally, this has meant bilateral exploration of the neck to identify all four parathyroids, followed by thorough assessment of the glands and removal of the abnormal glands. The most recently developed surgical approach involves the creation of a small unilateral incision to allow the insertion of surgical instruments, either alone or with a videoscope. ${ }^{13}$ Minimally invasive parathyroid surgery can include laparoscopic, radioguided, or unilateral neck surgery only. For minimally invasive surgery, precise preoperative localization is critical. Minimally invasive parathyroid surgery is appropriate only for patients who have a single, clearly defined parathyroid abnormality on ultrasound, sestamibi scan, or both, and when parathyroid hormone levels can be monitored intraoperatively. Bilateral neck exploration is mandatory in all other cases and for patients with familial or genetic syndromes. Compared with the conventional approach, minimally invasive parathyroidectomy is also cost-effective and, most importantly, results in a lower incidence of postoperative hypocalcemia. ${ }^{29,30}$ As shown by our four cases, serum parathyroid hormone levels decline rapidly after parathyroidectomy, while serum calcium normalizes over the course of several days. The correction of serum calcium and parathyroid hormone is accompanied by resolution of all symptoms of hypercalcemia. However, it is to be noted that follow-up of symptoms and associated conditions along with biochemical confirmation would be informative at six months. Normalization of serum calcium levels and resolution of all symptoms associated with hypercalcemia at six months are highly indicative of a curative parathyroidectomy. Research studies suggest that minimally invasive parathyroidectomy, when performed by experienced surgeons, offer a high success rate (95\%-98\%) and a low complication rate $(1 \%)$ due to recurrent laryngeal nerve injury. ${ }^{31}$

\section{Conclusion}

Hyperparathyroidism secondary to parathyroid tumors must be considered in the differential diagnosis for severe hypercalcemia in all age groups. The disease is often initially misdiagnosed because patients may present with a wide range of general symptoms. However, if no emergency treatment is provided, the case could result in severe and potentially fatal complications, ${ }^{32,33}$ thus underscoring the importance of early diagnosis followed by prompt surgical treatment. Furthermore, as observed in our case reports, the initial differentiation between benign parathyroid disease and malignancy is often complicated due to the similar clinical manifestations and relatively high serum calcium level. Preoperative imaging in the setting of primary hyperparathyroidism is mainly designed to assist the surgeon in identifying the anatomic localization of abnormally functioning or enlarged parathyroid glands. It also offers a possibility for a less invasive surgical approach. The use of calcitonin and biphosphonates for medical treatment and correction of hypercalcemia prior to surgery is also debatable due to their limited effect and the risk of postoperative hypocalcemia. Medical optimization is an important initial step providing an effective bridge to surgical treatment, but should definitely not delay the timing of surgery. Finally, this report supports the evidence that surgical management of patients presenting with hyperparathyroid crisis secondary to adenomatous disease is highly effective ${ }^{34}$ and provides the best outcome for the patient. Excision of the pathological parathyroid tumor results in rapid correction of serum calcium and parathyroid hormone levels, followed by gradual resolution of the metabolic consequences of severe hypercalcemia.

\section{Disclosure}

The authors report no conflicts of interest in this work.

\section{References}

1. Wermers RA, Khosla S, Atkinson EJ, et al. Incidence of primary hyperparathyroidism in Rochester, Minnesota, 1993-2001: An update on the changing epidemiology of the disease. J Bone Miner Res. 2006;21: 171-177.

2. Udelsman R. Six hundred and fifty-six consecutive explorations for primary hyperparathyroidism. Ann Surg. 2002;235:665-670.

3. Edelson GW, Kleerekoper M. Hypercalcemic crisis. Med Clin North Am. 1995;79:79-92.

4. Boonen S, Vanderschueren D, Pelemans W, Bouillon R. Primary hyperthyroidism. Diagnosis and management in the older individual. Eur J Endocrinol. 2004;151:297-304.

5. Silveberg SJ, Bilezikian JP. The diagnosis and management of asymptomatic primary hyperparathyroidism. Nat Clin Pract Endocrinol Metab. 2006;2:494-503.

6. Robert JH, Trombetti A, Garcia A, et al. Primary hyperparathyroidism: Can parathyroid carcinoma be anticipated on clinical and biochemical grounds? Report of nine cases and review of the literature. Ann Surg Oncol. 2005;12:526-532.

7. Kung B, Winokur R, Cognetti D, O'Hara B, Rosen D. Parathyroid carcinoma: Rare cause of primary hyperparathyroidism. Ear Nose Throat J. 2009;88:E10-E13.

8. Wang C, Gaz RD. Natural history of parathyroid carcinoma. Diagnosis, treatment and results. Am J Surg. 1985;149:522-527. 
9. Schantz A, Castleman B. Parathyroid carcinoma. A study of 70 cases. Cancer. 1973;31:600-605.

10. Johnson SJ. Changing clinicopathological practice in parathyroid disease. Histopathology. 2010;56:835-851.

11. Chan JK, Tsang WY. Endocrine malignancies that may mimic benign lesions. Semin Diagn Pathol. 1995;12:45-63.

12. Ito F, Sippel R, Lederman R, et al. The utility of intraoperative bilateral internal jugular venous sampling with rapid parathyroid hormone testing. Ann Surg. 2007;245:959-963.

13. Suliburk JW, Perrier ND. Primary hyperparathyroidism. Oncologist. 2007; 12:644-653.

14. Boggs JE, Irvin GL 3rd, Carneiro DM, Molinari AS. The evolution of parathyroidectomy failures. Surgery. 1999;126:998-1002.

15. McBiles M, Lambert AT, Cote MG, et al. Sestamibi parathyroid imaging. Semin Nucl Med. 1995;25:221-234.

16. Ruda JM, Hollenbeak C, Stack BC Jr. A systematic review of the diagnosis and treatment of primary hyperparathyroidism from 1995 to 2003. Otolaryngol Head Neck Surg. 2005;132:359-372.

17. Lumachi F, Zucchetta P, Marzola MC, et al. Advantages of combined technetium-99m-sestamibi scintigraphy and high-resolution ultrasonography in parathyroid localization: Comparative study in 91 patients with primary hyperparathyroidism. Eur J Endocrinol. 2000;143:755-760.

18. Solorzano CC, Carneiro-Pla DM, Irvin GL 3rd. Surgeon-performed ultrasonography as the initial and only localizing study in sporadic and primary hyperparathyroidism. J Am Coll Surg. 2006;202:18-24.

19. Ferlin G, Borsato N, Camerani M, Conte N, Zotti D. New perspectives in localizing enlarged parathyroids by technetium-thallium subtraction scan. J Nucl Med. 1983;24:438-441.

20. Johnson NA, Tublin ME, Ogilvie JB. Parathyroid imaging: Technique and role in the preoperative evaluation of primary hyperparathyroidism. AJR Am J Roentgenol. 2007;188:1706-1715.

21. Ziegler R. Hypercalcemic crisis. J Am Soc Nephrol. 2001;12 Suppl 17: S3-S9.

22. Kaiser W, Biesenback G, Kramar R, et al. Calcium free hemodialysis: An effective therapy in hypercalcemic crisis - report of 4 cases. Intensive Care Med. 1989;15:471-474.
23. De Papp AE, Kinder B, LiVolsi V, Gupta SM, Stewart AF. Parathyroid carcinoma arising from parathyroid hyperplasia: Autoinfarction following intravenous treatment with pamidronate. Am J Med. 1994;97:399.

24. Farese S. The hungry bone syndrome - an update. Ther Umsch. 2007; 64:277-280. German.

25. Mundy GR, Guise TA. Hypercalcemia of malignancy. Am J Med. 1997; 103:134-145.

26. Lourwood DL. The pharmacology and therapeutic utility of bisphosphonates. Pharmacotherapy. 1998;18:779-789.

27. Phitayakorn R, McHenry CR. Hyperparathyroid crisis: Use of bisphosphonates as a bridge to parathyroidectomy. J Am Coll Surg. 2008;206: 1106-1115.

28. Iihara M, Yamashita T, Tanaka R, et al. Seven patients with hyperparathyroid crisis: Emphasis on prompt parathyroidectomy. Nippon Naibunpi Gakkai Zasshi. 1993;69:1051-1056. Japanese.

29. Westerdahl J, Bergenfelz A. Unilateral versus bilateral neck exploration for primary hyperparathyroidism: Five-year follow-up of a randomized controlled trial. Ann Surg. 2007;246:976-980.

30. Russell CF, Dolan SJ, Laird JD. Randomized clinical trial comparing scan-directed unilateral versus bilateral cervical exploration for primary hyperparathyroidism due to solitary adenoma. Br J Surg. 2006;93: 418-421.

31. Irvin GL 3rd, Solorzano CC, Carneiro DM. Quick intraoperative parathyroid hormones assay: Surgical adjunct to allow limited parathyroidectomy, improves success rate, and predict outcome. World J Surg. 2004;28:1287-1292.

32. Lew JI, Solorzano CC, Irvin GL. Long-term results of parathyroidectomy for hypercalcemic crisis. Arch Surg. 2006;141:696-699.

33. Harjit K, Zanariah H, Hisham A. Hypercalcemic crisis. Immediate parathyroidectomy and intraoperative intravenous calcium infusion improves outcome. Asian J Surg. 2007;30:173-177.

34. Cannon J, Lew JI, Solórzano CC. Parathyroidectomy for hypercalcemic crisis: 40 years' experience and long-term outcomes. Surgery. 2010;148: 807-813.
International Medical Case Reports Journal

\section{Publish your work in this journal}

The International Medical Case Reports Journal is an international, peer-reviewed open-access journal publishing original case reports from all medical specialties. Previously unpublished medical posters are also accepted relating to any area of clinical or preclinical science. Submissions should not normally exceed 2,000 words or

\section{Dovepress}

4 published pages including figures, diagrams and references. The manuscript management system is completely online and includes a very quick and fair peer-review system, which is all easy to use. Visit $\mathrm{http}: / / \mathrm{ww} w$. dovepress.com/testimonials.php to read real quotes from published authors. 